\title{
Relief of acute cough by cough syrup with mucoprotection
}

\author{
Tobias Mück, PhD
}

Two randomised, blind, controlled, multicentre studies investigated the therapeutic effect of a chemical and mechanical barrier in the upper airways produced by polysaccharideresin-honey based cough syrup in children with acute cough associated with a common cold. The mucoprotection led to a significant alleviation of the paediatric cough compared with carbocysteine syrup or placebo. The beneficial effect was especially apparent in children with severe cough and/or episodes of nocturnal coughing.

$C_{\text {tom }}^{\text {ond }}$ ough, especially night-time cough, is one of the troublesome accompaniments to colds. Two studies illustrate how a chemical-mechanical barrier can protect the mucosa, promote its healing and thereby relieve the cough.

Cough symptoms in an acute cold are essentially triggered by a neuronal hypersensitivity of the cough reflex. Rhinoviruses, bacteria or irritants lead to inflammatory processes at the mucosal nerve endings and neuromodulation of the affected mucous membranes. If the mucosa can be protected from noxious substances and other harmful factors, it can recover and heal itself. Mucoadhesive substances - such as herbal polysaccharides or honey - that lie like a protective film on the mucosa, are suitable agents. In addition to providing physical protection, they have a hydrating effect, liquefy the sticky mucus and make it easier to cough up. As antioxidants, flavonoids bind free radicals that can develop in the body as a result of the infection.

A randomised, single blind multicentre study that appeared in the "World Journal of Pediatrics" investigated the effects of a mucoprotective cough syrup, the ingredients of which included herbal polysaccharides, honey and flavonoids, on cough in children [1]. 150 children aged between 2 and 5 years were included in the study that compared this syrup, a medicinal product with natural ingredients, with a carbocysteine syrup. The effects on daytime and nighttime cough accompanying an acute infection of the upper respiratory tract were considered. Particular attention was paid to any improvement in night-time cough. For this purpose, the night before starting the study medication was compared with night after it was begun. After only one day's use, there were significant differences between the herbal preparation and carbocysteine in relation to all parameters studied (see Figure). The former reduced the number of coughing fits about five times better than the comparator medication and the active syrup showed as much as a twelvefold better effect in terms of nocturnal coughing episodes.

A randomised, multicentre, double-blind and placebocontrolled study published in the "Italian Journal of Pediatrics" also investigated the efficacy of a cough syrup with natural molecular complexes [2]. The syrup, a medicinal product, contained herbal polysaccharides, honey and flavonoids. 102 children with upper respiratory tract infections and aged between three and six years old were enrolled in the study. The cough had lasted for at least seven days, but not more than three weeks. The effects of the active syrup were compared with that of a placebo syrup.

The active syrup was especially beneficial in children with a severe cough. In the subgroup of children with frequent or very frequent coughing episodes that caused marked impairment, the cough improved by Day 4 after the start of treatment in 13 of 14 children (93\%), but in only 7 of 13 children in the placebo arm $(54 \%)(p=0.03)$. Improvement was defined as no cough whatsoever to a maximum of two short coughing episodes of about ten minutes per day.

Summary: Both studies illustrate the positive effect of mechanical mucoprotection on the relief of paediatric cough associated with colds. The improvement in night-time cough is particularly notable. The protective film over the mucosa reduces contact with irritating substances as well as with microorganisms and thereby promotes the physiological regeneration. Use of the syrup is safe and effective and the

Evid Self Med 2021;1:210004 | https://doi.org/10.52778/efsm.21.0004

Affiliation/Correspondence: Tobias Mück, PhD, Consumer Healthcare Medical Affairs, Sanofi-Aventis Deutschland GmbH, Industriepark Hoechst, 65026 Frankfurt am Main, Germany (tobias.mueck@sanofi.com) 
0.2

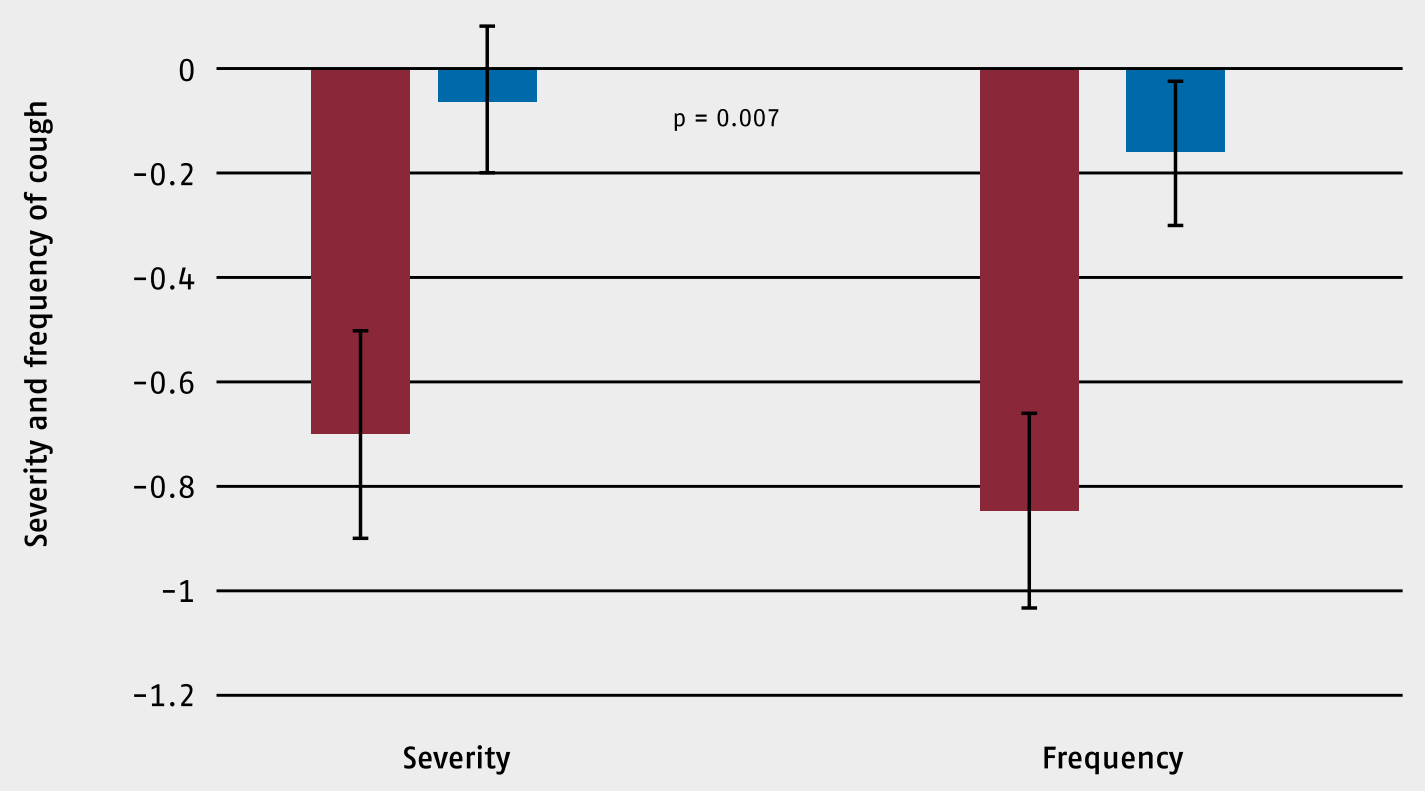

Fig.: Reduction in severity and frequency of the cough after 24 hours. Red: Active syrup (polysaccharide-resin-honey based cough syrup) $(n=75)$; Blue: Carbocysteine syrup $(n=66)$.

onset of action can be expected as early as the first day of treatment.

\section{Literature}

Cohen HA et al. World J Pediatr. 2017;13:27-33.

Canciani M et al. Italian Journal of Pediatr. 2014;40:56.
Conflict of interest: T. Mück is an employee of Sanofi.

Disclosure: Medical writing and publication funded by Sanofi Aventis Deutschland GmbH.

\section{Information regarding manuscript}

Submitted on: 17.09.2020

Accepted on: 26.12.2020

Published on: 16.08.2021 